\title{
Commentary on "Disclosure of funding source and conflict of interest: exposure of biases affecting evidence and clinical utility of plastic surgery publications" by Y. Tahiri et al.
}

\author{
Horacio F. Mayer
}

Received: 31 October 2011 /Accepted: 2 November 2011 /Published online: 28 December 2011

(C) Springer-Verlag 2011

The authors should be commended for conducting a study on the disclosure of funding source, conflict of interest, and level of evidence in plastic surgery publications. The European Journal of Plastic Surgery has recently established a mandatory financial disclosure process which will be published both online and in print with each manuscript. This is an important step to improve the transparency and integrity of our journal and its published articles.
This step will be continued through the introduction of evidence-based medicine (EBM) to the review process. Authors will be asked to assign an EBM value to their own papers and reviewers will be asked to rank every reviewed paper.

Although, the practice of EBM has been just recently introduced in our surgical specialty, plastic surgery research already seems to becoming more evidence based. Such change will certainly be translated into a rational and better clinical practice.
H. F. Mayer $(\bowtie)$

Department of Plastic Surgery, Hospital Italiano de Buenos Aires, University of Buenos Aires School of Medicine,

Buenos Aires, Argentina

e-mail: horacio.mayer@hospitalitaliano.org.ar 